\title{
Analysis of Nucleic Acid and Antibody Detection Results for SARS-CoV-2 Infection
}

\author{
Juanjuan Chen, MD ${ }^{1 *}$; Zhili Niu, MD ${ }^{1 *}$; Huan Li, MD'; Dongling Tang, MD'; Pingan Zhang, MD $^{1 *}$ \\ 'Department of Clinical Laboratory, Renmin Hospital of Wuhan University, Wuhan 430060, Hubei, China
}

\begin{abstract}
Background: Real-time polymerase chain reaction (RT-PCR) of virus nucleic acid test (NAT) has become the standard method to diagnose severe acute respiratory syndrome coronavirus 2 (SARS-CoV-2) infection. However, there are still many limitations, especially the problem of the high false negative rate. Therefore, the aim of this study was to investigate the positive rate of SARS-CoV-2 NAT and evaluate the diagnostic performance of SARS-CoV-2 IgM and IgG antibody detection in novel coronavirus infection.

Methods: A total of 10309 suspected or high-risk cases of infection with SARS-CoV-2 in Wuhan Hubei, China, were tested for virus NAT by RT-PCR. Among those cases, 762 COVID-19 patients and 143 patients with non-COVID-19 who were tested for SARS-CoV-2 IgM and IgG during the NAT period were screened. The difference between the two test methods was analyzed using the chi-square test.

Results: The positive rate of 10309 cases was about $36 \%$ (95\% Cl: $33.39 \%-39.67 \%)$. SARS-CoV-2 was present in various types of specimens, and alveolar lavage fluid had the highest positive rate $[52.38 \%(95 \% \mathrm{Cl}: 31.02-73.74)]$. The clinical sensitivity of serum SARS-CoV-2 IgM and IgG was $77.17 \%$ (588/762) and 94.88\% (723/762), respectively, and the clinical specificity was 93.71\% (134/143) and 90.21\% (129/143). The area under the curve (AUC) of SARS-CoV-2 IgG and combination of IgG with IgM were equally larger than IgM [0.973 (95\% Cl: 0.964-0.983) vs 0.930 (95\% Cl: 0.910-0.949)]. IgG antibody had the highest specificity [100.0\% (95\% Cl: $100.00 \%-100.00 \%)]$ and sensitivity [94.0\% (95\% Cl: $92.45 \%-95.55 \%)]$ when detected alone or in combination with IgM antibody. The total coincidence rate of SARS-CoV-2 antibodies detection and SARS-CoV-2 NAT for the diagnosis of SARS-CoV-2 infection was 92.04\% (833/905). Among the 34 SARS-CoV-2 NAT-negative patients with clinical symptoms and CT imaging features, 29 (85.29\%) patients were positive for SARS-CoV-2 IgM, and 31 (91.76\%) were positive for $\lg$.

Conclusion: SARS-CoV-2 NAT should be considered for many types of specimens, and the combined test of SARS-CoV-2 IgM and IgG can make up for the problem of missed NAT in COVID-19 patients.

Key words: Antibody, COVID-19, Detection, Nucleic acid, SARS-CoV-2 infection

Cite this article as: Chen J, Niu Z, Li H, Tang D, Zhang P. Analysis of Nucleic Acid and Antibody Detection Results for SARS-CoV-2 Infection. Arch Iran Med. 2021;24(5):427-433. doi: 10.34172/aim.2021.61
\end{abstract}

Received: April 17, 2020, Accepted: February 27, 2021, ePublished: May 1, 202

\section{Introduction}

Since December 2019, the coronavirus disease 2019 (COVID-19) emerged in Wuhan, Hubei, China, and subsequently a novel coronavirus (SARS-CoV-2) was confirmed to be the cause of COVID-19. ${ }^{1}$ The number of virus infections in China is increasing, and has exceeded 80000 by mid-March 2020. Early detection, early isolation and early therapy are the keys to epidemic prevention and control. $^{2,3}$ In addition, reducing secondary infections of close contacts and medical workers is also a focal point in preventing the spread of the disease.

However, distinguishing between COVID-19 patients and healthy individuals remains a major problem. For confirmed cases of COVID-19, the common clinical symptoms reported are similar to those of influenza virus infection, including fever, cough, fatigue, and muscle aches, ${ }^{4,5}$ which makes the diagnosis of COVID-19 more difficult. At present, the real-time polymerase chain reaction (RT-PCR) technology for SARS-CoV-2 nucleic acid test (NAT), CT imaging and clinical data are the main methods of diagnosing virus infection. ${ }^{6}$ Due to the severe epidemic situation in the Hubei province, the SARS-CoV-2 NAT of respiratory tract specimens, mainly nasal and pharyngeal swabs, has become the standard method to diagnose COVID-19 patients according to the recommendations for diagnosis and therapy of pneumonia caused by SARS-CoV-2 (the 5th edition). Yet, there are many limitations in the diagnostic criterion: (1) The problems with sampling; (2) RT-PCR requires a certified experimental platform and professional testing personnel; (3) The duration of the test may take up to three hours, and the process is complicated; (4) The RT-PCR of COVID-19 patients has a certain number of false negatives. ${ }^{7}$ Due to these limitations, RT-PCR is not suitable for rapid and convenient screening and diagnosis of infected patients. It slows the speed of epidemic prevention and control. Therefore, a method was developed for rapid detection of serum antibodies in order to quickly identify the infected 
person, prevent the continuous spread of the virus, and ensure that patients receive timely treatment. ${ }^{8}$

Detecting specific antigens or antibodies in patients' blood is the best choice for rapid, simple and highly sensitive clinical diagnosis of various diseases, such as tumors, viral infections and other diseases. ${ }^{9-11}$ At present, although a variety of antibody detection kits against SARS-CoV-2 virus have been developed, their clinical application value is rarely reported in the literature.

Therefore, the purpose of this study is to study the positive rate of SARS-CoV-2 NAT in various specimens and evaluate the laboratory diagnostic value of serum SARS-CoV IgM and IgG test in COVID-19.

\section{Patients and Methods}

\section{Study Population}

A total of 10309 cases with suspected or high-risk SARSCoV-2 infection were recruited for SARS-CoV-2 NAT or chest imaging and clinical symptom assessment in outpatient and inpatient settings in the Renmin Hospital of Wuhan University from January 21 to March 13 (Figure 1). All subjects either had typical respiratory tract infection symptoms, or had a history of close contact with SARSCoV-2-infected patients. Among those cases, 4523 were man (43.87\%) and 5786 were women (56.13\%), and they were aged from 22 to 98 years. Further, 905 subjects were recruited for the comparative analysis of the two detection methods, including 143 patients with diseases other than the new coronavirus pneumonia (68 men, 75 women, aged 24 to 100 years), and 762 COVID-19 patients (370 men, and 392 women including 728 patients with SARS-CoV-2
NAT positive, and 34 patients with virus NAT negative but with clinical symptoms and CT test results in line with the national diagnosis and treatment recommendations of COVID-19, 5th edition). SARS-CoV-2 IgM and IgG antibodies were tested in case groups and healthy controls.

\section{Diagnostic Criteria for SARS-COV-2 Infection}

(1) Respiratory tract or blood samples were positive for SARS-CoV-2 NAT by RT-PCR; (2) The viral gene sequence of the isolate from the respiratory tract or blood sample was highly homologous to the known new coronavirus.

\section{Sample Collection}

Respiratory or other specimens of all subjects were collected for SARS-CoV-2 NAT. Five milliliters of fasting venous blood was collected from the 762-case group and the 143-case group, placed in a yellow-head vacuum blood collection tube containing separation gel, and left to stand until the blood coagulated, centrifuged at the $2500 \times \mathrm{g}$ for 5 minutes, and serum was taken for use.

\section{Laboratory Analyses}

The open reading frame (ORF1ab) and nucleoprotein $(\mathrm{N})$ in the SARS-CoV-2 genome were tested by ABI ViiA7 real-time fluorescent quantitative PCR system, and the result was interpreted according to the reagent instructions. All supporting reagents were provided by Shanghai Huirui Biotechnology Co., Ltd. The result was diagnosed as positive if at least one target sequence in the same specimen was detected as positive by RT-PCR. We used commercial chemiluminescence detection kits

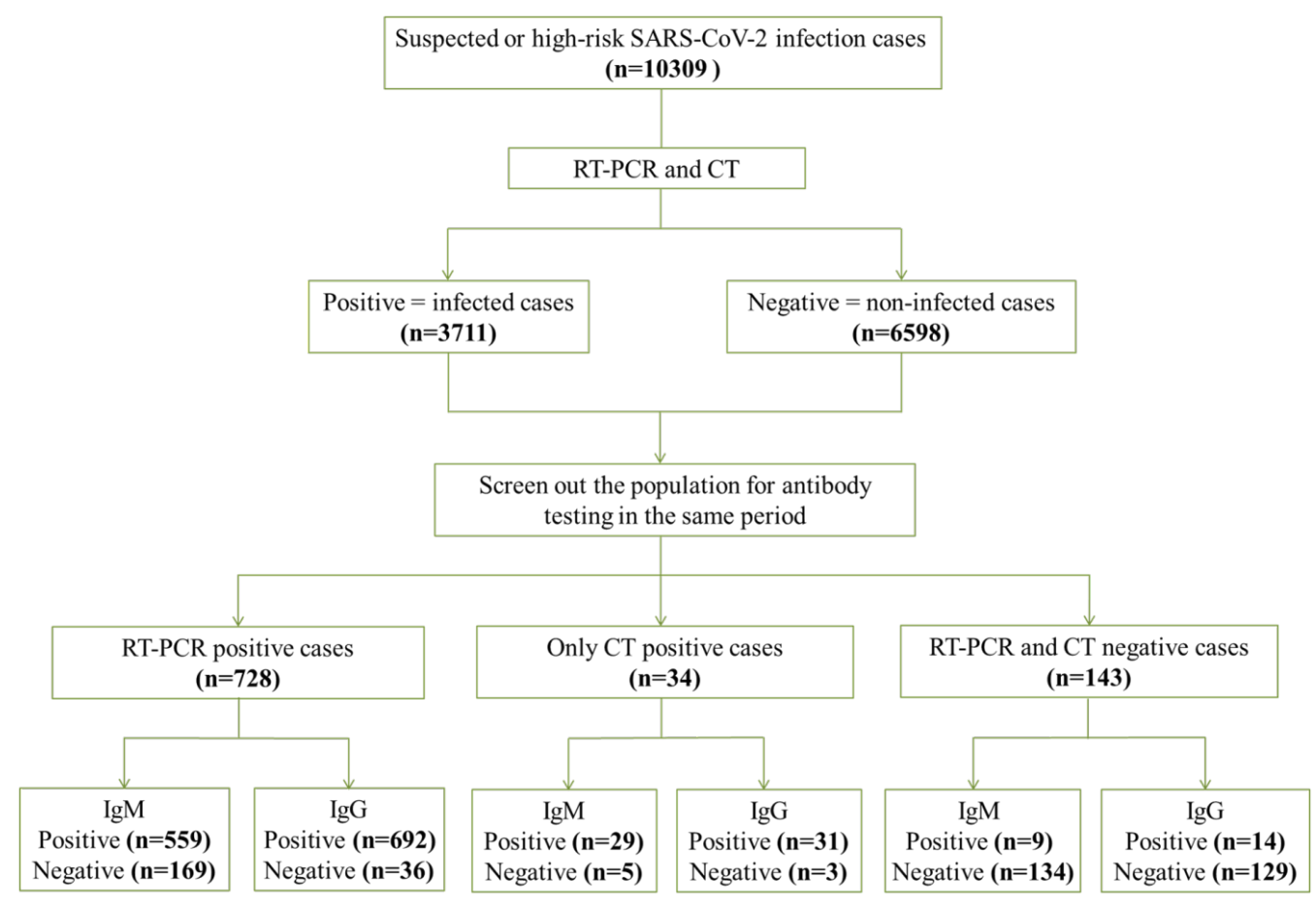

Figure 1. Study Flowchart. The screening and order of diagnostic testing of 10309 samples recruited. 
and IFlash3000 automatic chemiluminescent immune analyzer purchased from Shenzhen Yahuilong Biological Technology Co., Ltd to measure the levels of SARS-CoV-2 IgM and IgG in crude serum specimens, and results equal to or greater than $10.0 \mathrm{AU} / \mathrm{mL}$ were considered positive.

\section{Statistical Analysis}

The SPSS software version 20.0 (IBM, Armonk, NY) was used for statistical analysis of all data. The survey data were enumeration data, and the percentage and column list statistical methods were used. The paired chi-square test (McNemar test) was used for comparison of nucleic acid and antibodies test results. Receiver operating characteristic (ROC) curves were used to assess sensitivity, specificity, and area under the curve (AUC) within $95 \%$ confidence interval. The optimal cut-off value for diagnosis was determined by the maximum sum of sensitivity and specificity. The diagnostic accuracy of the combination of $\operatorname{IgM}$ and $\operatorname{IgG}$ was predicted using binary logistic regression and then the predicted value was used as a new marker for drawing the ROC curve. Finally, 2-sided $P$ values $<0.05$ were defined as statistically significant.

\section{Results}

Comparison of SARS-CoV-2 NAT Positive Rate in a Variety of Samples by RT-PCR

A total of 3766 out of 10309 cases (36.53\%, 95\% CI: $33.39 \%-39.67 \%)$ were positive for NAT on their respiratory samples or other types of specimens. Based on the detection and analysis of SARS-CoV-2 nucleic acid double-locus, the respiratory samples with the largest sample size were taken as the main observation object, and it was found that there were unit point and double-locus positivity, and the double positive rate was higher than the single positive rate (nasal and pharyngeal swabs): $26.33 \%$ (95\% CI: $25.48 \%-27.18 \%)$ vs $9.68 \%$ (95\% CI: $9.11 \%-$ 10.25\%); sputum: $27.14 \%$ (95\% CI: $23.61 \%-30.67 \%$ ) vs $12.83 \%$ (95\% CI: $10.17 \%-15.49 \%$ ); bronchoalveolar lavage fluid (BLF): 38.10\% (95\% CI: $17.33 \%-58.87 \%$ ) vs $14.29 \%$ (95\% CI:-0.67\%-29.26\%), as shown in Table 1. In addition, we found that the BLF showed the highest positive rate $(52.38 \%, 95 \%$ CI: $31.02 \%-73.74 \%)$, followed by sputum (39.97\%, 95\% CI: $36.08 \%-43.86 \%)$ and nasal and pharyngeal swabs (36.01\%, 95\% CI: $35.08 \%-36.94 \%)$. Remarkably, we also found SARS-CoV-2 in other specimens such as stool, anal swabs, urine, whole blood, breast milk, and eye secretions, and the positive rates were $12.13 \%, 9.48 \%, 6.00 \%, 7.14 \%, 33.33 \%$, and $3.22 \%$, respectively.

Results of SARS-CoV-2 NAT Positivity Status of Other Specimens in People with Negative Nasal and Pharyngeal Swabs

Among the 10309 subjects, 54 patients with negative nasopharyngeal swab showed positive results for the new coronavirus in other types of specimens, including 45 cases of sputum, 2 cases of BLF, 3 cases of stool, 1 case of urine, 1 case of breast milk, as seen in Table 2 .

\section{Clinical Specificity of SARS-CoV-2 IgM and IgG Antibody Test}

In the control group, 134 out of the 143 cases were negative for IgM, 9 cases were false positives, and the clinical specificity was $93.71 \%$ (134/143). SARS-CoV-2 IgG of 129 cases was negative, 14 cases were false positives, and the clinical specificity was $90.21 \%$ (129/143). Among 9 falsepositive controls of IgM antibody, 2 cases had higher IgM levels, up to $56.78 \mathrm{AU} / \mathrm{mL}$, of whom one case had received blood transfusion, and one case had an immune disease. The remaining seven cases were weakly positive and the results ranged from 10.50 to $23.27 \mathrm{AU} / \mathrm{mL}$; all of them were tumor patients. Among the 14 false positive SARSCoV-2 IgG antibody controls, the antibody level was in the range of $10.32 \mathrm{AU} / \mathrm{mL}$ to $16.49 \mathrm{AU} / \mathrm{mL}$, which showed a weak positive reaction.

\section{Clinical Sensitivity of SARS-CoV-2 IgM and IgG Antibody Test}

Among the 762 virus-infected patients, 588 were positive and 174 were negative for IgM antibody, 723 were positive for IgG antibody, and 39 were negative. The sensitivity of SARS-CoV-2 IgM and IgG was 77.17\% (588/762) and $94.88 \%$ (723/762), respectively.

\section{Diagnostic Ability of SARS-CoV-2 IgM and IgG Antibody for SARS-CoV-2 Infection}

We then assessed the diagnostic efficiency of serum IgM and IgG in patients with COVID-19 (Table 3). We drew the ROC curves of IgM and IgG for COVID-19 patients and disease controls. When the cut-off of IgM was set at $4.05 \mathrm{AU} / \mathrm{mL}$, we obtained an AUC of 0.930 (95\% CI: $0.910-0.949$ ), sensitivity of $89.00 \%$ (95\% CI: $86.96 \%-$ $91.04 \%$ ), and specificity of $87.4 \%$ (95\% CI: 85.24-89.56). When the cut-off of serum IgG was set at $16.86 \mathrm{AU} / \mathrm{mL}$, we obtained an AUC of 0.973 , sensitivity of $94.00 \%$ (95\%CI: 92.45\%-95.55\%), and specificity of $100.00 \%$ (95\% CI: $100.00 \%-100.00 \%)$. We further assessed the diagnostic ability of the combination of IgM and IgG, and found that the diagnostic value of the combined test was exactly the same as that of IgG alone (Figure 2).

\section{Comparison of SARS-CoV-2 Antibody and SARS-CoV-2 Nucleic Acid Detection}

The results of the detection of SARS-CoV-2 IgM and IgG antibodies in 905 subjects were compared with those of nucleic acid detection (Table 4 ). The positive predictive value of the antibody test was $93.97 \%(701 / 746)$, the negative predictive value was $83.02 \%(132 / 159)$, the positive predictive value of SARS-CoV-2 NAT was $100 \%$ $(728 / 728)$, and the negative predictive value was $80.79 \%$ $(143 / 177)$. The overall coincidence rate of SARS-CoV-2 antibody and NAT to diagnose SARS-CoV-2 infection was $92.04 \%$ (833/905). There were statistically significant differences between the two methods for diagnosing SARS-COV-2 infection $(\chi 2=4.50, P<0.05)$. Serological tests of 34 clinically diagnosed patients with SARS-CoV-2- 


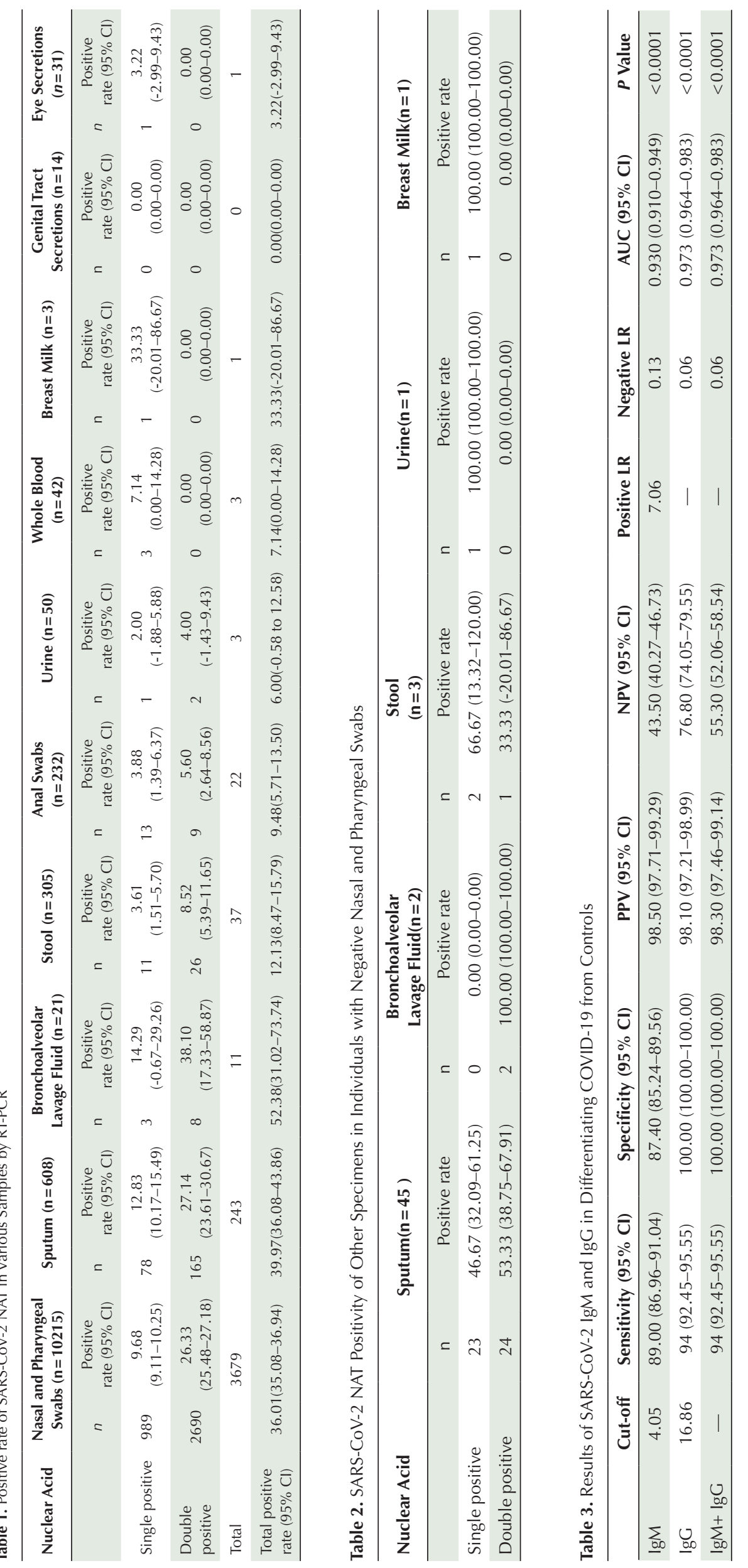




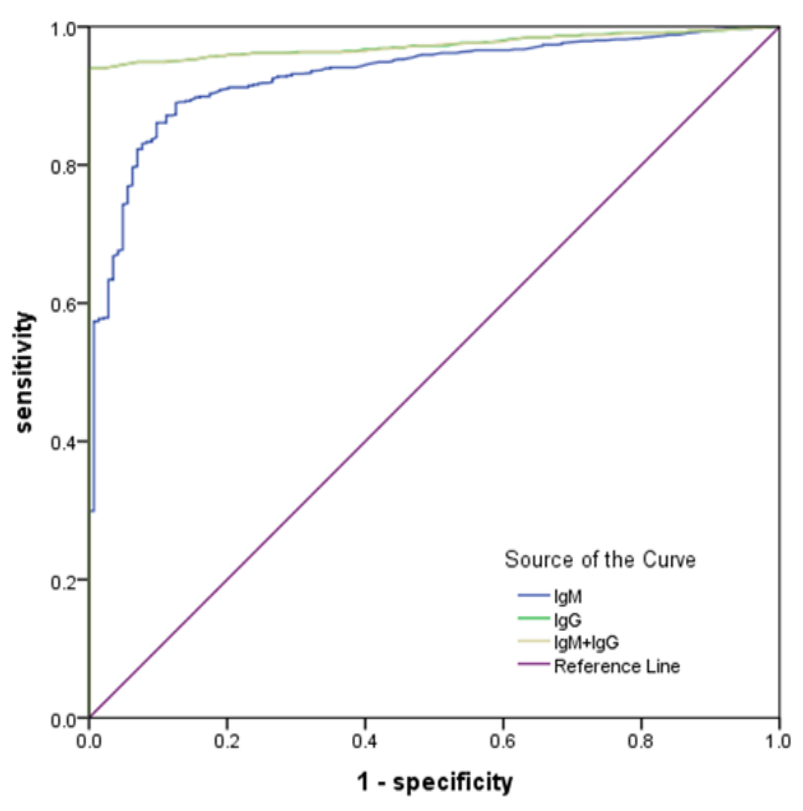

Figure 2. ROC curve of SARS-CoV-2 $\lg M$, $\lg G$ and the Combination for Diagnosing SARS-CoV-2 Infections.

infected symptoms and CT imaging features but negative NAT showed that $29(85.29 \%)$ of the 34 patients were positive for IgM antibody and $31(91.76 \%)$ were positive for IgG antibody.

\section{Discussion}

With the deepening of our knowledge about the clinical symptoms of SARS-CoV-2 infection, the characteristics of COVID-19 have shown some similarities with SARS, such as fever, chills and respiratory symptoms. ${ }^{12-14}$ Unlike the typical symptoms of SARS, some infected patients only had chills and respiratory symptoms or no symptoms of infection in the early stage, and even some patients with COVID-19 had gastrointestinal discomfort as the first symptom, which causes certain problems for the diagnosis of COVID-19. Therefore, the diagnosis of pneumonia caused by suspected SARS-CoV-2 infection in China at the moment was based on the updated national guidelines for diagnosis and therapy of pneumonia caused by SARS-CoV-2 (7th edition), specifically, clinical symptoms, chest imaging, SARS-CoV-2 NAT, additional pathological features and viral antibody, and the ruling out of pneumonia caused by other pathogens.

In the present study, 10,309 patients with suspected SARS-CoV-2 infection in a hospital in Wuhan were analyzed by RT-PCR, and the results showed that the total number of positives accounted for $36.53 \%$ (95\%CI: $33.39 \%-39.67 \%$ ), which is close to previous research results. ${ }^{15}$ The test results were single positive and double positive, and the double positive rate was higher than the single positive rate. After the classification and analysis of respiratory tract specimens, we found that most samples were collected from nasopharyngeal swabs (10,215 cases) for virus detection in respiratory tract specimens,
Table 4. Comparison of Detection Results of SARS-CoV-2 Antibodies and SARS-CoV-2 Nucleic Acid

\begin{tabular}{lcccc}
\hline & & \multicolumn{3}{c}{ SARS-CoV-2 NAT } \\
\cline { 3 - 5 } & & Positive & Negative & Total \\
\hline \multirow{2}{*}{$\begin{array}{l}\text { SARS-CoV-2 } \\
\text { antibody testing }\end{array}$} & Positive & 701 & 45 & 746 \\
& Negative & 27 & 132 & 159 \\
& Total & 728 & 177 & 905 \\
\hline
\end{tabular}

followed by sputum (618 cases), and finally BLF ( 21 cases), which had a strong relationship with the convenience of sampling. Of the three respiratory tract specimens, the highest positive rate pertained to BLF (52.38\%; 95\% CI: $31.02 \%-73.74 \%$ ), followed by sputum (39.97\%; $95 \%$ CI: $36.08 \%-43.86 \%$ ), and finally, nasopharyngeal swabs (36.01\%; 95\%CI: $35.08 \%-36.94 \%)$. The reason may be that the former two belong to the lower respiratory tract, closer to the lung infection, and the nasopharyngeal swab is taken from the upper respiratory tract, which is prone to negative results caused by the operation and sampling time. The above results suggest that the BLF samples may be more suitable as the sampling object for the detection of respiratory pathogens. When testing sputum and nasopharyngeal swabs, attention should be paid to taking samples multiple times to repeatedly confirm the correctness of the test results. Previous studies have shown that in addition to SARS-COV-2 virus examined in respiratory samples, SARS-COV-2 was also present in specimens such as stool, urine and whole blood, ${ }^{16,17}$ but our research found that anal swabs, breast milk, and eye secretions can also detect the virus, suggesting that SARSCOV-2 may have the ability to infect most systems of the human body, and its mode of transmission may be diverse. However, no study has confirmed that SARS-COV-2 could be spread via the fecal-oral or eye secretions routes, so these can only be potential transmission routes. ${ }^{16,18}$ Moreover, our study also found that although SARSCOV-2 NAT for nasopharyngeal swabs was negative in the diagnosis of multiple suspected infected persons, it yielded positive results in other specimens, such as sputum BLF, feces and urine, other systems fluids, and ocular secretion, which reminds clinicians to take as many types of samples as possible when diagnosing suspected SARSCOV-2 infections to avoid the potential transmission risk caused by false negative results.

Currently, SARS-CoV-2 infection is spreading rapidly around the world. Diagnosing infected patients as soon as possible and cutting off the transmission route are crucial for controlling the epidemic as soon as possible. Antibody testing is the most rapid auxiliary diagnostic tool in clinical testing. The antibody detection kit of SARS-CoV-2 has been gradually applied to the auxiliary examination of SARS-CoV-2 infection; however, as the antibody test is a novel detection method for SARS-CoV-2, it is necessary to fully verify the clinical sensitivity and specificity of the serological laboratory diagnosis of COVID-19, especially to study whether the serological detection of 
antigen antibody can play a significant complementary role in nucleic acid missed detection. This study showed that the IgM and IgG immunoassay reagents of SARSCoV-2 had good clinical specificity, approaching 93.71\% and $90.21 \%$ separately, and can fully meet the needs of clinical testing. The sensitivity of SARS-CoV-2 IgM to detect COVID-19 reached $77.17 \%$, while it was as high as $94.48 \%$ for the IgG test, which can be effectively used to screen and diagnose novel coronavirus. ROC curves showed that the diagnostic ability of IgG was better than that of $\operatorname{IgM}$, and the diagnostic value of the combined test is equivalent to that of IgG alone. In addition, our results showed that among the 34 COVID-19 patients whose nucleic acid was missed but confirmed based on clinical symptoms, 29 out of 34 patients (85.29\%) were positive for SARS-CoV-2 IgM, and 31 out of 34 patients (91.76\%) were positive for IgG, indicating that antibody testing can effectively compensate for the risk of missed nucleic acid detection, and holds a significant laboratory diagnostic value in the rapid and accurate diagnosis, treatment and prevention of COVID-19. Currently, there are several possible factors for the false negative result of SARSCoV-2 NAT, including the specimen quality problems, such as early or late collection of specimens, inappropriate transportation, storage methods, the operational and instrument problems in testing and the testing techniques themselves caused by virus mutation, etc. ${ }^{19}$ The samples for serological detection of antigens and antibodies come from the peripheral blood, serum or plasma specimens that are easy to collect and store, and antibodies have good stability in serum specimens, thus laying a foundation for their high clinical detection sensitivity. In addition, compared to RT-PCR, it also has the advantages of short detection cycle, easy operation and minimal training.

In conclusion, multiple types of specimens should be considered for NAT for the diagnosis of SARS-CoV-2 infection. Additional testing for serum SARS-CoV-2 IgM and IgG can be used as an effective screening and diagnostic indicator for SARS-CoV-2 infection, and is an effective complement to COVID 19 nucleic acid false negative test.

\section{Authors' Contribution}

NL offered the idea of this work. JC collected data and wrote the manuscript. PZ, DT, HL corrected and guided the manuscript. All authors reviewed and approved the final manuscript.

\section{Conflict of Interest Disclosures}

The authors have no conflicts of interest.

\section{Ethical Statement}

This study was reviewed and approved by the Medical Ethics Review Committee of Renmin Hospital of Wuhan University (WDRM2020-K030) and was approved to waive the patient's informed consent.

\section{Funding Sources}

This research was supported by the National Natural Science Foundation of China (grant numbers: 81773444) and Hubei Natural Science Foundation of China (grant number: 2019CFC846).

\section{References}

1. Zhu N, Zhang D, Wang W, Li X, Yang B, Song J, et al. A Novel Coronavirus from Patients with Pneumonia in China, 2019. N Engl J Med. 2020;382(8):727-33. doi: 10.1056/NEJMoa2001017.

2. Liu NN, Tan JC, Li J, Li S, Cai Y, Wang H. COVID-19 Pandemic: Experiences in China and implications for its prevention and treatment worldwide. Curr Cancer Drug Targets. 2020;20(6):410-6. doi: 10.2174/156800962066620 0414151419.

3. Gong M, Li L, Xin S, Yue Y, Wang S, Hong Z. CloudBased System for Effective Surveillance and Control of COVID-19: Useful Experiences From Hubei, China. J Med Internet Res. 2020;22(4):e18948. doi: 10.2196/18948.

4. Xu XW, Wu XX, Jiang XG, Xu KJ, Ying LJ, Ma CL, et al. Clinical findings in a group of patients infected with the 2019 novel coronavirus (SARS-Cov-2) outside of Wuhan, China: retrospective case series. BMJ. 2020;368:m606. doi: 10.1136/bmj.m606.

5. Wang C, Yu HJ, Horby PW, Cao B, Wu P, Yang SG, et al. Comparison of patients hospitalized with influenza A subtypes H7N9, H5N1, and 2009 Pandemic H1N1. Clin Infect Dis. 2014;58(8):1095-103. doi: 10.1093/cid/ciu053.

6. Jin YH, Cai L, Cheng ZS, Cheng H, Deng T, Fan YP, et al. A rapid advice guideline for the diagnosis and treatment of 2019 novel coronavirus (2019-nCoV) infected pneumonia (standard version). Mil Med Res. 2020;7(1):4. doi: 10.1186/ s40779-020-0233-6.

7. Pan Y, Long L, Zhang D, Yan T, Cui S, Yang P, et al. Potential false-negative nucleic acid testing results for Severe Acute Respiratory Syndrome Coronavirus 2 from thermal inactivation of samples with low viral loads. Clin Chem. 2020;66(6):794-801. doi: 10.1093/clinchem/hvaa091.

8. Li ZT, Yi YX, Luo XM, Xiong N, Liu Y, Li SQ, et al. Development and clinical application of a rapid IgMIgG combined antibody test for SARS-CoV-2 infection diagnosis. J Med Virol. 2020;92(9):1518-24. doi: 10.1002/ jmv.25727.

9. Ma J, Ren Y, He L, He X, Wenge X, Yan J. An efficient method for simultaneously screening for HIV, syphilis, and HCV based on one dried blood spot sample. Antiviral Res. 2020;181:104775. doi: 10.1016/j.antiviral.2020.104775.

10. Kori LD, Valecha N, Anvikar AR. Glutamate dehydrogenase: a novel candidate to diagnose Plasmodium falciparum through rapid diagnostic test in blood specimen from fever patients. Sci Rep. 2020;10(1):6307. doi: 10.1038/s41598020-62850-x.

11. Ye X, Li C, Zu X, Lin M, Liu Q, Liu J, et al. A Large-Scale Multicenter Study Validates Aldo-Keto Reductase Family 1 Member B10 as a Prevalent Serum Marker for Detection of Hepatocellular Carcinoma. Hepatology. 2019;69(6):2489501. doi: 10.1002/hep.30519.

12. Huang X, Wei F, Hu L, Wen L, Chen K. Epidemiology and Clinical Characteristics of COVID-19. Arch Iran Med. 2020; 23(4):268-71. doi: 10.34172/aim.2020.09.

13. Chen N, Zhou M, Dong X, Qu J, Gong F, Han Y, et al. Epidemiological and clinical characteristics of 99 cases of 
2019 novel coronavirus pneumonia in Wuhan, China: a descriptive study. Lancet. 2020;395(10223):507-13. doi: 10.1016/S0140-6736(20)30211-7.

14. Zhou F, Yu T, Du R, Fan G, Liu Y, Liu Z, et al. Clinical course and risk factors for mortality of adult inpatients with COVID-19 in Wuhan, China: a retrospective cohort study. Lancet. 2020;395(10229):1054-62. doi: 10.1016/S01406736(20)30566-3.

15. Liu R, Han H, Liu F, Lv Z, Wu K, Liu Y, et al. Positive rate of RT-PCR detection of SARS-CoV-2 infection in 4880 cases from one hospital in Wuhan, China, from Jan to Feb 2020. Clin Chim Acta. 2020; 505:172-5. doi: 10.1016/j. cca.2020.03.009.

16. Yeo C, Kaushal S, Yeo D. Enteric involvement of coronaviruses: is faecal-oral transmission of SARS-CoV-2 possible? Lancet Gastroenterol Hepatol. 2020;5(4):335-7. doi: 10.1016/S2468-1253(20)30048-0.

17. Chen Y, Chen L, Deng Q, Zhang G, Wu K, Ni L, et al. The Presence of SARS-CoV-2 RNA in Feces of COVID-19 Patients. J Med Virol. 2020;92(7):833-40. doi: 10.1002/ jmv.25825.

18. Yu AY, Tu R, Shao X, Pan A, Zhou K, Huang J. A comprehensive Chinese experience against SARS-CoV-2 in ophthalmology. Eye Vis (Lond). 2020;7:19. doi: 10.1186/ s40662-020-00187-2.

19. Xia X. Extreme genomic CpG deficiency in SARS-CoV-2 and evasion of host antiviral defense. Mol Biol Evol. 2020; 37(9):2699-705. doi: 10.1093/molbev/msaa094. 